Hanna Meissner

Universidade Técnica de Berlim, Berlim, Alemanha

\title{
La política como encuentro y respons-habilidad: aprender a conversar con los otros enigmáticos
}

Resumo: Partiendo de la pregunta de cuál podría ser la política de los nuevos materialismos feminis tas, este artículo contempla las posibilidades de (re)plantearla en términos de encuentros e implicación, de manera que ya no se basa en elegir y decidir, sino que es "el único modo en que crees que puedes seguir con vida" (Reagon, 1983). En nuestra época de dominio hegemónico antropocéntrico de lo político (Scott, 1999), veo aportaciones importantes de los nuevos materialismos (feministas) al desafío de replantearnos nuestros modos de relacionarnos con los "otros" (humanos y más que humanos), los cuales, sin necesariamente seguir las reglas, constituyen no obstante fuerzas agentivas. Reconocer nuestra dependencia fundamental como seres vivos enredados en mundos humanos y más que humanos ofrece la base ética para trabajar en modos de relacionarse con "otros" que aceptan e incluso adoptan el hecho de que nuestras certezas no permanecerán estables en tal proceso. Propongo interpretar el replanteamiento antifundacionalista que elabora Judith Butler (2011) de las nociones de intencionalidad y agencia política a través de la crítica de Karen Barad (2007), según la cual Butler solamente atribuye dinamismo e historicidad de la materia a la agencia del lenguaje o la cultura. Sugiero reanimar y perfilar el replanteamiento de la subjetividad política de Butler a través de la crítica de Barad (2007), cuando revisa la afirmación de Butler de que la materia es "aquello que provoca y ocasiona." Argumento que esta afirmación impide distinguir claramente entre pasividad y actividad, por lo que permite modificar nuestra comprensión de la subjetividad y agencia en términos de "estar con" y responder al tratamiento enigmático del otro (Basile, 2005).

Palabras clove: nociones de lo político, performatividad, nuevo materialismo, antropocentrismo, responsabilidad

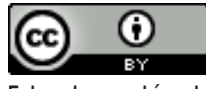

Esta obra está sob licença Creative Commons.

${ }^{1}$ Artigo publicado originalmente em Artnodes: Revista de Arte, Ciencia y Tecnologia, n. 14, 2014. (cc) Hanna Meissner.

${ }^{2}$ Aún ha de establecerse hasta qué punto y si está justificado hablar
¿Cuál es la política de los nuevos materialismos feministas? ${ }^{1},{ }^{2}$ ¿Y qué nociones nuevas de política emergen en estos debates? Estas cuestiones apremiantes aún han de responderse, y puede que nunca se respondan de manera definitiva, sino que más bien mantengan abierta e inestable cualquier noción de política y agencia política. Los nuevos materialismos feministas plantean desafíos importantes para redefinir nociones y prácticas que "nosotros" concebimos como "políticas." En concreto, cuestionan el engreimiento 
antropocéntrico que implica entender lo político como la cumbre de la excepcionalidad humana, como la capacidad de transformar y dar forma al mundo (social). Partiendo de estas preguntas y retos, este artículo examina las posibilidades de (re)plantear lo político en términos de encuentros e implicación, de manera que ya no se base en elegir y en decidir, sino en la necesidad.

No obstante, al abordar los desafíos de los nuevos materialismos relativos a la cuestión política, es necesario elaborar más la crítica del antropocentrismo, en concreto. Para distinguirla de otras agencias y fuerzas transformadoras, la política es, por definición, una noción antropocéntrica que implica agentes humanos específicos que se esfuerzan por dar forma y transformar las condiciones en las que viven. Esto a su vez implica que los agentes políticamente activos sean sujetos quienes, hasta cierto punto, son conscientes de sus necesidades y motivos particulares, y a la vez tienen voluntad política e intenciones políticas. Estos sujetos pueden fijarse objetivos y definir los medios para conseguirlos. Es más, las condiciones de posibilidad de tales trans(formaciones) políticas vienen dadas por la suposición de que el mundo está constituido socialmente. La acción política es la actividad de los sujetos que perciben los problemas como problemas sociales y asumen que poseen la capacidad y puede que también el deber de dar forma activamente al mundo en el que viven para resolver o hacer que tales problemas disminuyan.

Los nuevos materialismos no comulgan con el antropocentrismo de tales suposiciones, por lo que cuestionan las presunciones y certezas que se dan por sentadas en lo político. El enfoque social como dimensión políticamente dócil de la realidad se percibe como una retirada de la materialidad, como una falta de atención a la agencia e historicidad de lo material. Los nuevos materialismos se esfuerzan por volver a articular la noción de políica como vinculación con la materia. Sin embargo, no está tan claro como puede parecer a primera vista a qué remite realmente el término materia, $\mathrm{ni}$ quién se vincula con tal materia, ni en qué condiciones lo hace. Como argumentaré más adelante, es importante reconocer el carácter situado de "nuestras" nociones de lo político para volver a imaginarlas desde dentro y contrapuestas.

\section{Situar la noción de política}

La cuestión de quién se vincula con la materia y en qué condiciones lo hace señala la necesidad de tener en cuenta otras intervenciones críticas que han reflexionado sobre la noción de lo que es político. Como han mostrado los críticos postcoloniales, la genealogía de la racionalidad política 
moderna no puede trazarse sin tener en cuenta los encuentros imperialistas. Tal y como argumenta David Scott, los regímenes coloniales implantaron reglas específicas en "un nuevo juego politico" (SCOT, 1999, p. 45), un juego que obligaba a cualquiera que aspirara a la acción política a seguirlas. Es más: parte de las reglas del juego exigían invalidar, de manera sistemática, otras formas de vida "desmontando sistemáticamente sus condiciones y estableciendo otras en su lugar para permitir - es decir, para imponer - que se desarrollaran nuevas formas de vida" (SCOT, 1999, p. 26). La presunción de que nuestro mundo puede organizarse, transformarse y crearse activamente, la cual constituye la base de nuestra noción de lo político, está profundamente arraigada en estas condiciones históricas. El poder moderno se basa fundamentalmente en la organización descentralizada de las condiciones de vida y, como nos han enseñado los análisis de Michel Foucault, el sujeto que se determina a sí mismo, que experimenta deseos e intenciones innatas como origen de la agencia política (potencialmente racional) resulta esencial en esta organización descentralizada (FOUCAULT, 1982). El poder moderno se basa en la producción de sujetos y el gobierno de su conducta, para lograr condiciones en las que esos sujetos se esfuercen por gobernarse a sí mismos de formas particularmente productivas. Parte de este régimen de poder en particular se impulsa en transformar y definir globalmente las condiciones de la vida y las subjetividades: "El problema político del poder colonial moderno era [...] no solamente contener la resistencia y animar a acomodarse" sino intentar garantizar que ambas solo pudieran definirse respecto a las categorías y estructuras de las racionalidades políticas modernas" (SCOTT, 1999, p. 52).

Para los proyectos referidos a la rearticulación de las nociones y prácticas de la política, esto significa que, paradójicamente, la subjetividad moderna es tanto el objeto de la crítica como el modo de resistirse a las relaciones de poder. Esta forma histórica de subjetividad configura el marco contemporáneo de inteligibilidad de lo humano; es esencial para las nociones jurídicas de derechos, para las reivindicaciones políticas y se involucra constitutivamente en la formación de la sociedad civil. Las condiciones de posibilidad para la agencia política y la acción transformadora están estructuradas por los regímenes modernos de saber y poder. La crítica postcolonial converge con la crítica del nuevo materialismo en el desafío de reconocer y explicar el rol constitutivo de los "otros", de quienes no pueden o no quieren acatar reglas hegemónicas de lo político, de quienes, pese a no seguirlas, son fuerzas agentivas. En nuestra época de reglas hegemónicas y globalizadas de lo político, "nosotros" tenemos que replantearnos cómo relacionarnos con "otros" marginados 
o incluso abyectos como formas contemporáneas de estar en el mundo, de crear formas específicas de vida, de subjetividad ética y de sociabilidad. Al comentar brevemente los debates postcoloniales en un artículo centrado en los desafíos que plantea el nuevo materialismo para la noción de lo político, pretendo resaltar que la distinción entre "humano" y "no humano" no es la única que está en juego en los desafíos críticos planteados a tal noción. O más bien, que si nos centramos exclusivamente en distinguir entre "humano" y "no humano", nos arriesgamos a reinstaurar nociones eurocéntricas de humanidad y de subjetividad humana, al equipararlas implícitamente con "lo humano." Si no se especifica claramente quiénes (¿"nosotros"?) problematizan el dualismo entre "humano" y "no humano", la falta de especificación implica una noción universal de la humanidad que, sin darse cuenta, restaura el humanismo centrado en Occidente (SCHUELLER, 2009, p. 237) que pretende derrocar.

Esto implica que hemos de tener cuidado con el "uso natural del 'nosotros'" (AHUJA, 2010, p. 131) y al mismo tiempo reconocer que no podemos limitarnos a abandonar la subjetividad que este "nosotros" implica. En cierto modo, nosotros, quienes debatimos estas cuestiones en el contexto académico de la modernidad tardía, estamos atrapados porque somos y acompañamos a ese sujeto humano. Vivimos en un mundo formado, en muchos sentidos, por agentes que pretenden actuar como seres humanos racionales que se esfuerzan por saber, dar forma e incluso controlar su entorno (pasivo). Las presunciones de dominio tecnológico no son solo ilusiones, sino que poseen efectos materiales potentes, se han materializado en la ontología histórica de nuestro presente y han pasado a formar parte del tejido vivo de nuestro ser material. Estos efectos materiales se abordan a través de los intentos políticos de transformación, y subjetividad humana de la modernidad tardía constituye "nuestro" conjunto de condiciones para que tal agencia transformadora se desarrolle.

Puede que se haya expuesto un argumento levemente fatalista para justificar por qué necesitamos aferrarnos, al menos de manera provisional, a ciertas suposiciones relativas a la subjetividad humana. Existe, no obstante, una línea de razonamiento emancipadora, que asume las tradiciones del materialismo histórico con su compromiso político de hacer visible, o accesible, el hecho de que la actividad humana constituye una fuerza positiva en la constitución de la realidad. Marx elaboró este argumento en su análisis del capitalismo para que fuera concebible que ciertas estructuras constituidas por el modo de producción capitalista fueran efectos de prácticas humanas, y por lo tanto pudieran transformarse mediante la agencia humana cooperativa. Su objetivo era desplazar las explicaciones naturalistas del trabajo, los 
recursos, las racionalidades económicas y demás para abrir el camino de la agencia emancipadora. Esta insistencia en que el modo capitalista de producción es un efecto de la agencia humana y por lo tanto puede transformarse a través de las prácticas humanas sigue resultando una necesidad histórica en nuestra época. Supone un contrapunto necesario al dogma neoliberal de que los procesos económicos eluden el conocimiento humano y deberían dejarse en manos de la dinámica del mercado (y naturalizarse en ella) (MIROWSKI, 2010).

\section{Una imagen más concurrida}

Así que nos enfrentamos a una especie de dilema. Nos encontramos y pertenecemos a una situación histórica en la que tenemos que aferrarnos al sujeto humano para cuestionar radicalmente ese mismo sujeto. Como dijo Donna Haraway: "Creo que 'nosotros' - esa construcción material y retórica decisiva de la política y la historia - necesitamos algo llamado humanidad. Es la clase de cosa que Gayatri Spivak denominaba "aquello que no podemos no querer" (HARAWAY, 2004, p. 49).

Este enredo con y en lo que criticamos implica que tenemos que plantearnos la rearticulación de la política como proceso desde dentro: trasformar las condiciones a través de los medios proporcionados por tales condiciones. Así, entendemos la política como performatividad, como políica desde dentro y contrapuesta (BUTLER y ATHANASIOU, 2013). La noción de performatividad es un concepto fundamental en el diálogo de Karen Barad con la obra de Judith Butler, en concreto en sus intentos de llevar los debates más allá del ámbito de la agencia humana. A Butler le interesan sobre todo los sujetos humanos, o, para ser más precisos, los modos en que los límites humanos definen la noción de sujeto humano y constituyen sujetos particulares, que marginan y excluyen a otros. Barad critica que Butler se centre en la subjetividad humana y nos insta a ir más allá de lo humano. Pero, tal y como la interpreto, Barad no abandona la noción de sujetos humanos como tales, sino que fuerza el concepto de performatividad humana para mostrar "una imagen mucho más concurrida, en la cual es improbable que [los sujetos humanos] sean el único elemento a analizar" (BELL, 2012, p. 22).

Mientras Butler se concentra en las posibilidades de replantearse la intencionalidad y la agencia (política) en una descripción posthumanista de la subjetividad humana, Barad señala nuevas posibilidades de mostrarse receptivo con "otros" rechazando que la noción de agencia se restrinja a la intencionalidad o la subjetividad humana. El argumento crucial de Barad es que "la materia tiene un papel agentivo en su materialización iterative" (BARAD, 2007, p. 177). Barad 
critica que Butler "se centra exclusivamente en cuerpos humanos y factores sociales, lo cual va en contra de sus esfuerzos por entender la relación indisociable entre materialidad y discursividad" (BARAD, 2007, p. 34).

Según interpreta Barad, al centrarse en cuerpos humanos y factores sociales, Butler "en última instancia vuelve a inscribir la materia como producto pasivo de prácticas discursivas, en vez de como agente activo que participa en el proceso mismo de materialización" (BARAD, 2007, p. 151). Para justificar su afirmación, Barad remite a un pasaje de Cuerpos que importan en el que Butler propone que la materialidad del cuerpo "es una demanda en y por el lenguaje, 'aquello' que provoca y ocasiona, [que exige que] se lo explique, se lo describa, se lo diagnostique, se lo altere o [... . se lo alimente, se lo ejercite, se lo movilice, se lo adormezca, un sitio de actuaciones y pasiones de diversa índole. [...]. No la pizarra en blanco o el medio pasivo sobre el cual actúa la psique, sino, antes bien, la demanda constitutiva que moviliza la acción psíquica desde el comienzo" (BUTLER, 201 1, p. 108-109).

Barad concede que Butler ofrece una alternativa a las teorías del constructivismo social cuando enfatiza la importancia del exterior constitutivo, de aquello que tiene que excluirse para lograr la inteligibilidad discursiva. Sus planteamientos críticos señalan el problema de explicar el papel agentivo de la materialidad, centrándose en la dimensión lingüística de los procesos de materialización. Barad razona que "aunque Butler demanda correctamente el reconocimiento de la historicidad de la materia, resulta irónico que parezca asumir que en última instancia deriva (una vez más) de la agencia del lenguaje o la cultura. No logra reconocer el dinamismo de la materia" (BARAD, 2007, p. 65).

Butler está realmente interesada en la agencia constitutiva del lenguaje, pero no niega la posibilidad de otras agencias. En particular le interesan las agencias corporales y psíquicas de lo inaceptable, el dinamismo de la presencia espectral de aquello que está excluido del orden de inteligibilidad. Tal y como la interpreto en este contexto, lo que señala es que "nuestras" posibilidades de reconocer estas dinámicas están configuradas y constreñidas por el lenguaje. Entonces, la pregunta que se plantea a continuación es cómo podemos reelaborar nuestro enfoque conceptual de los dominios de lo ininteligible para aprender a tener conversaciones reales con "otros" ininteligibles.

Butler se centra claramente en el aparato lingüístico que participa en la constitución de los cuerpos como cuerpos sexuados. No obstante, no veo que en su argumentación tenga que haber una dicotomía entre actividad y pasividad o causa y efecto. De hecho, su afirmación - que la materialidad puede conceptualizarse como "una demanda en y 
por el lenguaje, 'aquello' que provoca y ocasiona" (BUTLER, 2011 , p. 108-109) - confunde toda distinción clara entre pasividad y actividad. "Provocar y ocasionar" puede considerarse una actividad, un "impacto provocador de un otro enigmático" (BASILE, 2005, p. 17). Butler piensa adoptando el punto de vista de un sujeto humano, pero intenta conceptualizar los modos según los cuales este sujeto se ve abordado y animado por "otros" que no lo son necesariamente. En un texto reciente, Butler remite a la noción de occasion según Alfred North Whitehead y la describe como una interacción curiosa: "Dado que tanto el sujeto como el objeto se animan al relacionarse el uno con el otro, algunas dimensiones de cada uno se suscitan porque se abordan mutuamente, y, en este sentido, la vitalidad de cada uno depende de cierta provocación procedente del otro" (BUTLER, 2012, p. 4). Así, se añade otra capa a la obra de Butler referida a la relacionalidad fundamental y a la dependencia constitutiva, que la lleva más allá de los confines de las relaciones humanas. Su obra permanece conceptualizada según el punto de vista humano o según el punto de vista de un mundo estructurado humanamente (BUTLER, 2012, p. 5), pero está claro que contiene la idea de que sujeto y objeto no pueden separarse de manera definitiva. Estamos inmersos, enredados constitutivamente, en mundos más que humanos; actuamos "mientras actúan sobre nosotros" (BUTLER, 2012, p.8). Como concluye Butler:

\begin{abstract}
si hubiera que replantearse la performatividad en estos términos, sería importante intentar entender el modo extraño en que actúan sobre nosotros, nos abordan, nos sacan, nos provocan, y cómo lo que denominamos nuestra "actuación" o nuestro "hacer" ya supone siempre, en ciertas maneras, una respuesta a lo que precede y permite nuestra acción. La teoría performativa de la acción tiene que volver a situarse dentro de una comprensión relacional de los organismos vivos, tanto humanos como no, para entender tanto lo que sustenta la vida como lo que la hace peligrar (BUTLER, 2012, p. 16).
\end{abstract}

\title{
El riesgo de la respons-habilidad
}

El riesgo de la respons-habilidad indica más posibilidades de diálogo con la obra de Karen Barad respecto a cómo rearticular nuestra comprensión de la performatividad y la responsabilidad sin limitarlas al sujeto humano: "La responsabilidad no es solo nuestra. [...] La responsabilidad implica una receptividad continuada a los enredos de uno mismo y del otro, aquí y allí, ahora y entonces" (BARAD 2007, p. 394). Por lo tanto, la responsabilidad (suma de responsabilidad y capacidad de respuesta ha de formularse, ya que la connotación clave ya no es el imperative de hacerse cargo y dar 
explicaciones, sino, más bien, la habilidad de responder a "otros". La responsabilidad se replantea como el mandamiento ético de desarrollar la capacidad de responder a "otros", de encargarnos de los enredos de nuestras relacionalidades. Esto implica que la respons-habilidad está vinculada a procesos de volverse distinto en y a través de la respuesta. Esta noción de responsabilidad implica una solidaridad que no se basa en la proximidad y la similitud, sino en la idea de que estamos metidos en esto todos juntos". Como señaló Bernice Johnson Reagon en su presentación en el Festival de Música de Mujeres de la Costa Oeste de 1981, el siglo xx está marcado por desarrollos tecnológicos que comportan la "posibilidad de asegurarse de que ningún ser humano quede fuera de su alcance", que conducen a "el fin de una era en la que tener un espacio que sea 'solo tuyo', solo para la gente que quieras que esté en él” (REAGON, 1983, pág. 357).

Considerando nuestra perspectiva a comienzos del siglo xxi, esta imposibilidad de aislar sociedades humanas (aparentemente) homogéneas aún se complica más, ante la imposibilidad de distinguir claramente a los individuos humanos como separables de un entorno no humano. Reconocer la dependencia constitutiva que nos genera nuestra existencia enredada con y en "otros" humanos y más que humanos nos insta a replantearnos el concepto de agencia. También nos insta a replantearnos lo político como proceso abierto de aprender y desaprender (SPIVAK, 1985), lo cual también implica aceptarlo como fracaso necesario para llegar a alcanzar algún objetivo definitivo. De manera destacada, lo político ya no se basa en elegir y decidir, sino en conectarse, en encuentros e implicación: "No formas una coalición solo porque te guste. El único motivo por el que te plantearías asociarte con alguien que posiblemente podría matarte es porque es el único modo que se te ha ocurrido de seguir con vida" (REAGON, 1983, pág. 356-357).

En un mundo de interdependencia global no podemos evitar estar metidos en esto con "otros". Tenemos que reconocer que, en nuestro presente histórico, estas relaciones de estar metidos en esto son, en muchos sentidos, jerárquicas y violentas de manera sistemática. Al concebir esta violencia en términos de relaciones de poder social, se puede imaginar la agencia política como una fuerza transformadora para lograr "un mundo mejor, un mundo habitable, un modo basado en valores de prosperidad conjunta y reciprocidad (BARAD, 2011 , pág. 450)". Pero el replanteamiento necesario de lo político implica un replanteamiento de la actividad transformadora. Reagon señala sucintamente el riesgo que implica tal política: "La mayor parte del tiempo te sientes amenazado hasta lo más hondo, y si no es así, es que no te estás fusionando realmente" (REAGON, 1983, pág. 356). 
Replantearse lo político implica prepararse para cuestionar radicalmente quiénes somos y qué podemos ser como sujetos humanos. No se trata de una tarea cómoda - sí de un mandamiento ético al que los nuevos materialismos añaden nuevas capas -, pero es una necesidad.

\section{Referencias}

AHUJA, Neel. "Rhetorics of Endangerment: Cultural Difference and Development in International Ape Conservation Discourse." En: ROOS, B.; HUN, A. (eds.). Postcolonial Green: Environmental Politics and World Narratives. Charlottesville: University of Virginia Press, 2010, p. 118-134.

BARAD, Karen. Meeting the universe halfway: Quantum physics and the entanglement of matter and meaning. Durham: Duke University Press, 2007.

"Erasers and Erasures: Pinch's Unfortunate 'Uncertainty Principle'." Social Studies of Science, v.41, n. 3, p. 443454, 2011.

BASILE, Elena (2005). "Responding to the Enigmatic Address of the Other: A Psychoanalytical Approach to the Translator's Labour." New Voices in Translation Studies. v. 1, p. 12-30, 2005.

BELL, Vikki. "After Performativity: On Concern and Critique." En: FABER R.; HALEWOOD M.; LIN D. (eds.). Butler on Whitehead: On the Occasion. Plymouth: Lexington Books, 2012, p. 19-28.

BUTLER, Judith. Bodies That Matter: On the Discursive Limits of 'Sex'. Nueva York: Routledge, 2011.

. "On this Occasion." En: FABER, R.; HALEWOOD M.; LIN D. (eds.). Butler on Whitehead: On the Occasion. Plymouth: Lexington Books, 2012, p. 3-17.

BUTLER, Judith; ATHANASIOU, Athena. Dispossession: The Performative in the Political. Cambridge: Polity Press, 2013.

COOLE, Diana. "Agentic Capacities and Capacious Historical Materialism: Thinking with New Materialisms in the Political Sciences." Millenium: Journal of International Studies, v. 0, n. 0, p. 1-19, 2013.

FOUCAULT, Michel (1982). "The Subject and Power." En DREYFUS, H. L.; RABINOW, P. (eds.). Michel Foucault: Beyond Structuralism and Hermeneutics. Chicago: The University of Chicago Press, 1982, p. 208-226.

HARAWAY, Donna (2004). “Ecce Homo, Aint (Ar'n't) I a Woman, and Inappropriate/d Others: The Human in a Post-Human Landscape." En: The Haraway Reader. Nueva York: Routledge, 2004, p. 47-61.

MIROWSKI, Philip. Never Let a Serious Crisis Go to Waste. How Neoliberalism Survived the Financial Meltdown. Londres: Verso, 2010.

REAGON, Bernice Johnson (1983). "Coalition Politics: Turning 
the Century." En: SMITH, B. (ed.). Home Girls: A Black Feminist Anthology. Nueva York: Kitchen Table/Women of Color Press, 1983, p. 356-368.

SCHUELLER, Malini Johar. "Decolonizing Global Theories Today." Interventions. v. 11 , n. 2, p. 235-254, 2009.

SCOTT, David. Refashioning Futures: Criticism after Postcoloniality. Nueva Jersey: Princeton University Press, 1999.

SPIVAK, Gayatri Chakravorty. "Criticism, Feminism and the Institution: An Interview with Gayatri Chakravorty Spivak.". Thesis Eleven, n. 10-1 1, p. 175-187, 1985.

TUIN, Iris van der (2011). "New Feminist Materialisms. Review Essay." Women's Studies International Forum, v. 34, n. 4, p. 271-277, 2011.

[Recebido em 01/08/2017 e aprovado em 12/01/2017]

Politics as Encounter and Response-Ability: Learning to Converse with Enigmatic Others Abstract: Starting from the question of what the politics of new feminist materialisms could be, this article addresses the possibilities of (re-)conceptualizing the political in terms of encounters and involvedness, but not foremost as a matter of choice and decision but as "the only way you can figure you can stay alive" (Reagon, 1983). In our times of hegemonic anthropocentric rule of the political (Scott, 1999), I see important contributions of new (feminist) materialisms to the challenge of reconsidering our modes of encountering "others" (human and more-than-human), who, without necessarily playing by the rules, are nevertheless agentive forces. Acknowledging our fundamental dependency as living beings enmeshed in human and more-than-human worlds provides ethical grounds for working on modes of encountering "others" that accept and even embrace the fact that our own certainties will not remain stable in the process. I propose a reading of Judith Butler's anti-foundationalist rethinking of humanist notions of intentionality and political agency (2011) through Karen Barad's critique of her attribution of matter's dynamism and historicity solely to the agency of language or culture (2007). I suggest that Butler's rethinking of political subjectivity can be re-invigorated and sharpened, in light of Barad's critique (2007), by revisiting Butler's claim that matter is "a 'that which' which prompts and occasions". I argue that this confounds any clear distinction of passivity and activity, thereby enabling a transformation of our understanding of subjectivity and agency in terms of being-with and responding to the enigmatic address of the other (Basile, 2005).

Keywords: notions of the political, performativity, new materialism, anthropocentrism, responsibility

Hanna Meissner (hanna.meissner@tu-berlin.de) é assistente pós-doutora no Center for Women's and Gender Studies (ZIFG) of the Technische University Berlin. Suas principais áreas de pesquisa são teoria feminista, teoria social, estudos de ciência e estudos póscoloniais. Publicou, recentemente, o capítulo "Opening Space: Learning to Un-learn Certainties as Limitations" em Teaching with Feminist Materialisms (Peta Hinton and Pat Treusch (Eds.). Athena: Utrecht University, 2015). 